\title{
HADRONIC DEGREES OF FREEDOM
}

\author{
MARCO TRAINI \\ Dipartimento di Fisica \\ Università degli Studi di Trento \\ I-38050 POVO (Trento), Italy \\ and \\ INFN G.C. Trento \\ E-mail: traini@science.unitn.it
}

\begin{abstract}
I report on the research activities performed under the (italian) MURST-PRIN project "Fisica Teorica del Nucleo e dei sistemi a piú corpi" covering part of the topics on hadronic degrees of freedom. The most recent achievements in the field are summarized focusing on the specific role of the nuclear physics community.
\end{abstract}

\section{Introduction}

The point of view I take to summarize some of the research activities in Italy in the field of hadronic physics, is determined by the (today accepted) fundamental degrees of freedom of the strong interactions, namely quarks and gluons. The Sec. 2 is devoted to the link of the fundamental theory of strong interactions, Quantum Chromodynamics (QCD), with effective degrees of freedom often used to study specific problems, in particular those degrees of freedom that played a peculiar and historical role in the investigation of hadronic systems: the constituent quarks and the related quark models. In Secs. 3 and 1 I discuss recent results on the electromagnetic interactions with hadrons at low energy studied within non-relativistic and relativistic quark models, respectively. Sec. 5 is devoted to the discussion of the electroweak structure of the nucleon and Sec. 6 to deep inelastic scattering. Conclusive remarks are drawn in Sec. 7 .

\section{QCD and effective degrees of freedom}

Why does the non-relativistic quark model (NRQM) reproduces at the quantitative level several hadron properties? And why - by an appropriate choice of the parameters - also several other models, totally different from NRQM (e.g., the chiral bag model) often fit the data? In a series of papers Morpurgo and Dillon $\mathrm{H}_{2}$ tried to answer to the previous question by using an original approach called Geperal parametrization method. Following the Morpurgo's review paper of ref.t, I summarize the approach in the case example of the

final: submitted to World Scientific on November 13, 2018 
baryon masses.

\subsection{General parametrization: basic ideas}

The QCD mass $M_{\mathrm{B}}$ of the baryon $\mathrm{B}$ is the expectation value of the exact QCD Hamiltonian, $H_{\mathrm{QCD}}$ on the exact lowest state $\Psi_{\mathrm{B}}$ of $\mathrm{B}$ :

$$
M_{\mathrm{B}}=\left\langle\Psi_{\mathrm{B}}\left|H_{\mathrm{QCD}}\right| \Psi_{\mathrm{B}}\right\rangle \text {. }
$$

One can write

$$
\left|\Psi_{\mathrm{B}}\right\rangle=\mathcal{V}\left|\phi_{\mathrm{B}}\right\rangle
$$

where the auxiliary state $\left|\phi_{\mathrm{B}}\right\rangle$ is a three-quark-no-antiquark-no-gluon state in Fock space and it reduces to the simplest NRQM.

Whereas $\left|\phi_{\mathrm{B}}\right\rangle$ is quite simple, $\mathcal{V}$ is exceedingly complicated. Indeed $\mathcal{V}$ must take into account:

i) the dress of the auxiliary state $\left|\phi_{\mathrm{B}}\right\rangle$ with $q \bar{q}$ pairs and gluons in order to transform it into the exact state which assumes the form:

$$
\left.\left|\Psi_{\mathrm{B}}\right\rangle=|q q q\rangle+|q q q, \bar{q} q\rangle+\mid q q q, \text { Gluons }\right\rangle+\ldots ;
$$

ii) the mixing of $S U_{3}$-flavor configurations;

iii) the Foldy-Wouthuysen transformation of the quark spin states from static 4 -spinors (with upper components $(0,1)$ or $(1,0))$ ) to Dirac 4-spinors.

\subsection{Baryon masses}

By using the $\mathcal{V}$ transformation, Eq.(11) becomes

$$
M_{\mathrm{B}}=\left\langle\Psi_{\mathrm{B}}\left|H_{\mathrm{QCD}}\right| \Psi_{\mathrm{B}}\right\rangle=\left\langle\phi_{\mathrm{B}}\left|\mathcal{V}^{\dagger} H_{\mathrm{QCD}} \mathcal{V}\right| \phi_{\mathrm{B}}\right\rangle \text {. }
$$

As final result one gets an expression for the "parametrized mass" operator which depends on the flavor and spin operators of the three quarks, namely:

$$
\begin{aligned}
\hat{\mathcal{M}}= & M_{0}+B \sum_{i} P_{i}^{S}+C \sum_{i>k}\left(\vec{\sigma}_{i} \cdot \vec{\sigma}_{k}\right)+D \sum_{i>k}\left(\vec{\sigma}_{i} \cdot \vec{\sigma}_{k}\right)\left(P_{i}^{S}+P_{k}^{S}\right)+ \\
& +E \sum_{i \neq k \neq j}\left(\vec{\sigma}_{i} \cdot \vec{\sigma}_{k}\right) P_{j}^{S}+a \sum_{i>k} P_{i}^{S} P_{k}^{S}+b \sum_{i>k}\left(\vec{\sigma}_{i} \cdot \vec{\sigma}_{k}\right)\left(P_{i}^{S} P_{k}^{S}\right)+ \\
& +c \sum_{i \neq k \neq j}\left(\vec{\sigma}_{i} \cdot \vec{\sigma}_{k}\right)\left(P_{i}^{S}+P_{k}^{S}\right) P_{j}^{S}+d P_{1}^{S} P_{2}^{S} P_{3}^{S}
\end{aligned}
$$


where $P_{i}^{S}$ are projection operators selecting the strange $S$-quark, and $\vec{\sigma}$ the quark spin Pauli matrices; $M_{0}, B, C, \ldots d$ are parameters and only the combination $a+b$ is relevant for the masses. Few comments on the general parametrization (5) are in order:

i) it has been obtained for the baryon masses but can be extended to many physical quantities of the lowest multiplets of hadrons (both baryons and mesons), like magnetic moments, semileptonic matrix elements etc..

ii) it shows a large similarity with the NRQM result, but it is exact within QCD and fully relativistic (even if non-covariant because derived in the rest frame of the nucleon). In particular it takes into accont the flavor breaking term $\Delta m \bar{\Psi} P^{S} \Psi$ in the QCD Lagrangian to all orders.

In order to better understand the approach, let me introduce a provocative question: the masses of the lowest octect and decuplet baryons are eight $(N, \Lambda, \Sigma, \Xi, \Delta(1232), \Sigma(1385), \Xi(1530)$ and $\Omega)$ and Eq.(5) contains eight parameters $\left(M_{0}, B, C, D, E, a+b, \mathrm{c}, \mathrm{d}\right)$ : why is it convenient to deal with the eight parameters rather than with the eight masses directly?

The key point is that the dynamical consequence of QCD produces a hierarchy of the paramenters which depends on the number of the indices in Eq.(5). Some of them result to be larger than others, a peculiarity absent in the conventional $S U_{3}$ group-theoretical parametrization of the flavor-breaking terms. Specifically one gets (the values are in $\mathrm{MeV}$ ):

$M_{0}=1076, B=192, C=45.6, D=-13.8 \pm 0.3,(a+b)=-16.0 \pm 1.4$, $E=5.1 \pm 0.3, c=1.1 \pm 0.7, d=4 \pm 3$.

Omitting all terms beyond $E$ in Eq.(5) (that is keeping first order flavorbreaking terms only) one obtains the Gell-Mann-Okubo mass formula.

The coefficients decrease for terms with increasing number of indices and, at equal number of indices, they decrease increasing the order in flavor breaking (that is the number of $P^{S}$ factors). Each additional $P^{S}$ factor implies a reduction of $\approx 0.3$ (e.g. the ratio $D / C$ ). For each additional index (at equal number of $P^{S}$ factors) the reduction is $\approx 0.37$ (e.g. the ratio $E / D$ ).

An example is given by the Coleman-Galshow ( $\mathrm{CG}$ ) mass formula

$$
p-n=\Sigma^{+}-\Sigma^{-}+\Xi^{-}-\Xi^{0},
$$

derived assuming unbroken $S_{3}$ flavor. Since flavor is violated (in the baryon octect) by $\approx 33 \%$, a similar violation of $\mathrm{CG}$ is expected. However by using the new recent measured vale of $\Xi^{0}, 1314 \pm 0.06 \pm 0.2 \mathrm{MeV}$, one gets, for the left and right side of Eq. (6), the impressive values 1.h.s. $=-1.29 \mathrm{MeV}$; r.h.s. $=-1.58 \pm 0.25 \mathrm{MeV}$.

The general parametrization approach is able to solve the mistery of the spectacular precision of the CG mass formulal. As matter of fact the expres- 
sion (6) is valid to all orders in flavor breaking terms with the omission of the terms with 3-quarks indices. The hierarchy in the general parametrization expansion allows an estimate of the relative contribution of the three-quark terms: $(1 / 3)^{3} \approx 4 \cdot 10^{-2}$ to be compared with the level of precision of the CG mass formula $((1.58-1.29) \pm 0.25) / 8 \approx(4 \pm 3) \%$, since the mass difference $\Sigma^{-}-\Sigma^{+} \approx 8 \mathrm{MeV}$. The present case is a peculiar case where, thanks to the hierarchy in the parametrization, an estimate of an effect due to strong interaction can be given and found tiny as expected.

\section{NRQM: electromagnetic transitions}

A consequence of the large success of the Constituent Quark Models (CQM) is the proliferation of a large class of different approaches.

In particular various models have been proposed for the internal baryon structure. A quite common feature of many models is that, despite of the use of different ingredients, they are able to give a satisfactory description of the baryon spectrum and, in general, of the nucleon static properties (cfr. the discussion of the previous section).

The obvious reason is that the study of hadron spectroscopy is not sufficient to distinguish among the various forms of quark dynamics and other observables, such as the electromagnetic transition form factors and the strong decay amplitudes, are important in testing models for the intefnal structure of the hadrons. As an example I discuss a recent calculation 3 of the electromagnetic transition form factors using different potential models within a specific approach which includes three-body forces within an hypercentral approximation.

\subsection{Transition form factors and the hypercentral potential model}

The electromagnetic transition form factors, $A_{1 / 2}\left(Q^{2}\right)$ and $A_{3 / 2}\left(Q^{2}\right)$, are defined as the transition matrix elements of the transverse electromagnetic interaction, $H_{e . m .}^{t}$, between the nucleon, $N$, and the resonance, $B$, states:

$$
A_{J_{z}^{\prime}}\left(Q^{2}\right)=\left\langle B, J^{\prime}, J_{z}^{\prime}\left|H_{e m}^{t}\right| N, J=1 / 2, J_{z}=1 / 2\right\rangle
$$

Following Aiello et al., I compare various models:

1) a potential which retains only the hypercoulomb and the linear confinement terms fitted to the spectrum, plus a standard hyperfine interaction;

2 ) an analytical model which corresponds to the previous approximation plus a hyperfine interaction with a sooth $x$-dependence;

final: submitted to World Scientific on November 13, 2018 


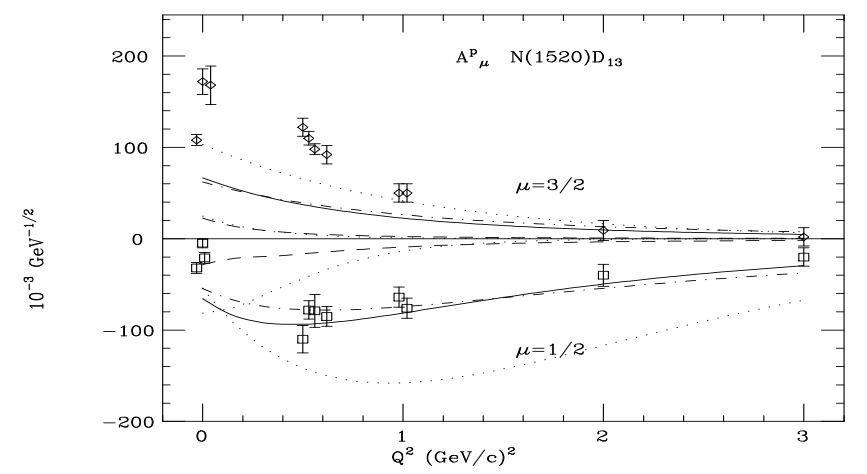

Figure 1. Comparison between the experimental data for the transition form factors $A_{3 / 2}^{p}, A_{1 / 2}^{p}$ for the $D_{13}(1520)$-resonance and the calculations with the potentials 1 ) (full curve), 2) (dot-dashed curve), 3) (dashed curve), 4) (the dotted curve with the stronger damping) and 5) (the dotted curve with the softer damping).

3) a potential which has the property of reproducing exactly the dipole fit of the proton form factor;

4) the harmonic oscillator with the parameter $\alpha=0.229 \mathrm{GeV}$ which reproduces the proton charge radius;

5) the harmonic oscillator with the parameter $\alpha=0.410 \mathrm{GeV}$ corresponding to a confinement radius of the order of $0.5 \mathrm{fm}$, required in order to reproduce the $A_{3 / 2}^{p}$ at $Q^{2}=0$ for the $D_{13}(1520)$-resonance.

In Fig. 11 the proton helicity amplitudes for $D_{13}(1520)$-resonance. The potentials 1) and 2) give rise to similar results. They both fit the energy levels and lead to a confinement radius of the order of $0.5 \mathrm{fm}$. The medium $Q^{2}$-behaviour is good but they fail to reproduce well the data at low $Q^{2}$ especially in the $A_{3 / 2}^{p}$ case.

The results are however very different from potential 1) and 2) and in the $A_{1 / 2}^{p}$ case also far from the data. The potential which reproduces exactly the dipole form factor, 3), gives too damped results; the same happens for the h.o. with the correct proton radius, which causes a too strong damping in the wave functions.

Similar conclusions can be drawn from Fig. 2, where the results for the $S_{11}(1535)$-resonance are shown. One can see that reproducing the elastic form 


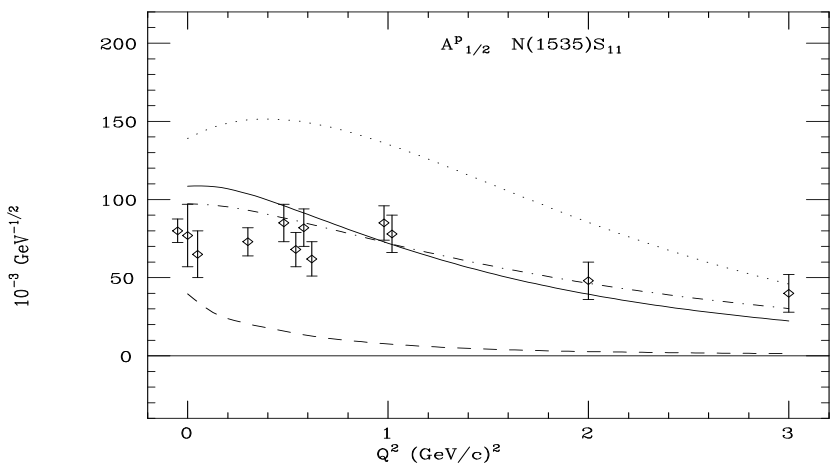

Figure 2. As in previous Figure, for the $A_{1 / 2}^{p}$ of the $S_{11}(1535)$-resonance, calculated with the potentials 1), 2), 3) and 5).

factor is not a guaranty for describing also the transition form factors. None of the CQM considered can explain adequately the transition form factors at low momentum transfer. The discrepancy indicates that some important effect at low momentum transfer is missing, like polarization effect of the Dirac sea, not included in CQMs. The calculations, at variance with what expected, are in agreement with the few existing data at $Q^{2}=1-2(\mathrm{GeV} / \mathrm{c})^{2}$, that is outside the range of applicability of a non relativistic description.

The problem of a relativistic description is still opent and I discuss relativistic extensions in Sec.A

\subsection{Inelastic photon scattering and the magnetic moment of the $\Delta$ (1232)}

As further example of CQM application let me briefly discuss inelastic photon scattering and its relation with the magnetic moment of the $\Delta(33)$ resonance as investigated by Drechsel et al. in ref.E.

The static properties of baryons are an important testing ground for QCD based calculations in the confinement region. However, little experimental information is available for hadrons outside of the ground state $\mathrm{SU}(3)$ octet. In view of the short life-time of the resonances, such information has to come from a detailed analysis of intermediate states. As a result of many experimental and theoretical efforts, the Particle Data Group (1998) quotes a value 
of $\mu_{\Delta^{++}}=(5.6 \pm 1.9) \mu_{N}$ for the magnetic dipole moment of the $\Delta^{++}$resonance. The large error bar is due to large nonresonant processes, external bremsstrahlung by initial and final state particles, and a strong background due to interactions in both the initial and final states. A much cleaner experiment would be an electromagnetic excitation of the nucleon leading to the $\Delta$ resonance with subsequent emission of a real photon followed by the decay into a nucleon and a pion. The process $\gamma+p \rightarrow \gamma^{\prime}+p^{\prime}+\pi^{0}$ would be particularly favorable, because the signal is less disturbed by the external bremsstrahlung background.

Unfortunately, the A2 collaboration at MAMI working with the TAPS detector has only been able to see 3 -photon events at a rate corresponding to a cross section of tens of nanobarns. Drechsel et al. point out that one only expect total cross sections for this process in the range of 5-10 nb, which is probably at the limit of the present experimental accuracy. For this purpose they have calculated both elastic (Compton) and inelastic photon scattering through the $\Delta$ resonance in the constituent quark model. The expectations based on such a simple model show that the integrated cross section for this process should indeed be very small, namely of the order of $5 \mathrm{nb}$.

The electromagnetic moments of baryon resonances are among the most evasive properties of hadrons. The extremely weak signals for these moments are at the very limits of even the most advanced experimental techniques. However, such data would be invaluable for our understanding of QCD in the confinement region, and dedicated experiments are certainly desirable.

\section{Covariant Quark Models: electromagnetic form factors}

In the present section I discuss the relativistic extension of CQM. In particular a relativistic light-front (LF) constituent-quark (CQ) medel and the investigation of transition electromagnetic hadron form factors 6 in the momentum transfer region relevant for the experimental research programme at TJNAF. The main features of the model are: i) eigenstates of a mass operator which reproduces a large part of the hadron spectrum; ii) a one-body current operator with phenomenological Dirac and Pauli form factors for the CQ's. The CQ's are assumed to interact via the $q-q$ potential of Capstick and Isgur (CI), which includes a linear confining term and an effective one-gluon-exchange (OGE) term. The latter produces a huge amount of high-momentum components in the baryon wave functions and contains a central Coulomb-like potential, a spin-dependent part, responsible for the hyperfine splitting of baryon masses, and a tensor part. A comparable amount of high momentum components was obtained with the $q-q$ interaction based on the exchange of the pseudoscalar 
Goldstone-bosons. This fact suggests that the hadron spectrum itself dictates the high momentum behaviour in hadron wave functions as furtherly suggested by the recent calculations of eleftromagnetic static form factors within the Goldstone-bosons potential modell. In the following I review results for the transition form factors for $J \leq 3 / 2$ hadrons.

\subsection{Nucleon-Resonance transistion form factors}

Once the $C Q$ form factors have been determined by means of the evaluation of elastic form factors within the LF model, one can obtain parameter-free predictions for the nucleon-resonance transition form factors.

In Fig. 3 the evaluations of the helicity amplitude $A_{1 / 2}$ are shown for $N \rightarrow S_{11}(1535), S_{11}(1650)$ and $S_{11}(1620)$, and compared with the results of the non-relativistic model of ref. $\mathrm{b}$. In the case of $S_{31}(1620)$ the results for $p$ and $n$ coincide (as for $P_{33}(1232)$ ), since only the isovector part of the CQ current is effective. The predictions yield an overall agreement with available experimental data for the $P$-wave resonances and show a sizeable sensitivity to

relativistic effects, but more accurate data are needed to reliably discriminate between different models.

\section{Electroweak structure of the nucleon}

In this section I review the research activity aimed to reveal the electroweak structure of the nucleon, in particular its strange content. I discuss both neutrino and parity-violating polarized electron scattering.

\subsection{The strange axial current}

After the measurements of the polarized structure function of the proton, $g_{1}$, in deep inelastic scattering, it turned out, rather surprisingly, that the constant $g_{A}^{s}$, that characterizes the one-nucleon matrix element of the axial strange current, namely

$$
\left\langle p, s\left|\bar{q} \gamma^{\alpha} \gamma_{5} q\right| p, s\right\rangle=2 M s^{\alpha} g_{A}^{q} ; \quad(\mathrm{q}=\mathrm{s}),
$$

is of magnitude comparable with the corresponding $g_{A}^{u}$ and $g_{A}^{d}$ axial constants. (Here $p$ is the nucleon momentum, $M$ is the nucleon mass, $s^{\alpha}$ is the spin vector and $g_{A}^{q}$ is a constant). A theoretical analysis of deep inelastic data led to the following values for the axial constants: $g_{A}^{s}=-0.10 \pm 0.03, g_{A}^{d}=$ $-0.43 \pm 0.03, g_{A}^{u}=0.83 \pm 0.03$ (in a more recent analysis of the data, the value $g_{A}^{s}=-0.13 \pm 0.03$ was reported). 

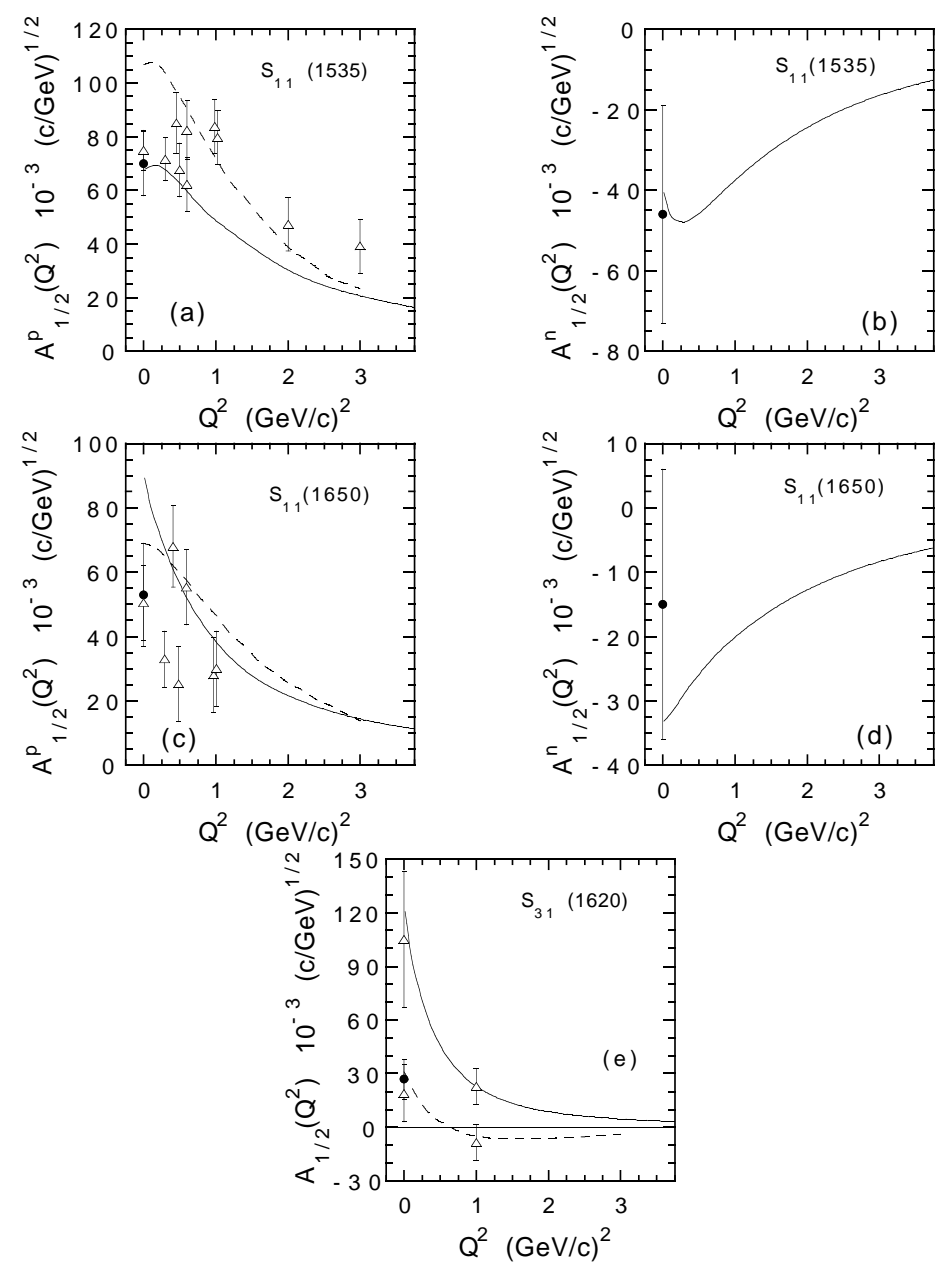

Figure 3. The transverse helicities $A_{1 / 2}^{p(n)}$ for the nucleon transitions $p \rightarrow S_{11}$ (1535) (a); $n \rightarrow S_{11}$ (1535) (b); $p \rightarrow S_{11}(1650)$ (c); $n \rightarrow S_{11}(1650)$ (d); $p \rightarrow S_{31}(1620)$ (e), vs. $Q^{2}$. The non relativistic calculations of ref. [3] are shown by the dashed lines.

However the values of the constants $g_{A}^{q}$ given of Eq.(8) were obtained under several assumptions. It is then clear that it is very important to use other methods for the determination of the matrix elements of the strange current. The investigation of neutral-current (NC) as well as charged-current 
(CC) neutrino reactions is one of these ways 0.10.

\subsection{The BNL-734 experiment}

Let one consider the elastic scattering of muon neutrinos and antineutrinos on nucleons: $\nu_{\mu}+N \rightarrow \nu_{\mu}+N ; \quad \bar{\nu}_{\mu}+N \rightarrow \bar{\nu}_{\mu}+N$.

The cross sections of these processes depend on the electromagnetic form factors, on the axial form factor and on the strange axial and vector form factors of the nucleon, and the value of the constant $g_{A}^{s}$ that can be extracted strongly depends on the behaviour of the (poorly known) axial form factor. In order to minimize this dependence Alberico et al. 9 consider the asymmetry

$$
\mathcal{A}_{p}\left(Q^{2}\right)=\frac{\left(\frac{d \sigma}{d Q^{2}}\right)_{\nu p \rightarrow \nu p}-\left(\frac{d \sigma}{d Q^{2}}\right)_{\bar{\nu} p \rightarrow \bar{\nu} p}}{\left(\frac{d \sigma}{d Q^{2}}\right)_{\nu n \rightarrow \mu^{-} p}-\left(\frac{d \sigma}{d Q^{2}}\right)_{\bar{\nu} p \rightarrow \mu^{+} n}}
$$

in order to obtain direct model independent information on the axial $\left(F_{A}^{s}\right)$ and magnetic $\left(G_{M}^{s}\right)$ strange form factors of the nucleon.

In ref. 0 the contribution of the strange form factors of the nucleon to the $\mathrm{NC}$ over $\mathrm{CC}$ neutrino-antineutrino asymmetry have been calculated and compared with the information on it, which one can extract from the data of the BNL-734 experiment. In this experiment the following ratios of cross sections were obtained:

$$
\begin{aligned}
R_{\nu} & =\frac{\langle\sigma\rangle_{(\nu p \rightarrow \nu p)}}{\langle\sigma\rangle_{\left(\nu n \rightarrow \mu^{-} p\right)}}=0.153 \pm 0.007 \pm 0.017 \\
R_{\bar{\nu}} & =\frac{\langle\sigma\rangle_{(\bar{\nu} p \rightarrow \bar{\nu} p)}}{\langle\sigma\rangle_{\left(\bar{\nu} p \rightarrow \mu^{+} n\right)}}=0.218 \pm 0.012 \pm 0.023 \\
R & =\frac{\langle\sigma\rangle_{(\bar{\nu} p \rightarrow \bar{\nu} p)}}{\langle\sigma\rangle_{(\nu p \rightarrow \nu p)}}=0.302 \pm 0.019 \pm 0.037
\end{aligned}
$$

where $\langle\sigma\rangle$ are cross sections folded with the experimental neutrino energy spectrum and integrated over the available range of momentum transfer $Q^{2}$.

The neutrino-antineutrino folded integral asymmetry, $\left\langle\mathcal{A}_{p}\right\rangle$, is also obtained from the neutral current to charge current ratio of the differences between the total folded neutrino and antineutrino cross sections 10 :

$$
\left\langle\mathcal{A}_{p}\right\rangle=\frac{\langle\sigma\rangle_{(\nu p \rightarrow \nu p)}-\langle\sigma\rangle_{(\bar{\nu} p \rightarrow \bar{\nu} p)}}{\langle\sigma\rangle_{\left(\nu n \rightarrow \mu^{-} p\right)}-\langle\sigma\rangle_{\left(\bar{\nu} p \rightarrow \mu^{+} n\right)}}=\frac{R_{\nu}(1-R)}{1-R R_{\nu} / R_{\bar{\nu}}}
$$


where the asymmetry has been written in terms of the ratios (10)-(12). From the experimental data one found:

$$
\left\langle\mathcal{A}_{p}\right\rangle=0.136 \pm 0.008 \text { (stat) } \pm 0.019 \text { (syst) } .
$$

In Fig. 4 the effects of strangeness for the ratios (10)-(12) and the integral asymmetry (13) are shown. The experimental values for the various quantities are indicated by the shadowed regions: the error band corresponds to one standard deviation. The usual dipole parameterization both for non-strange and strange form factors is assumed

$$
\begin{gathered}
F_{A}^{s}\left(Q^{2}\right)=g_{A}^{s} G_{D}^{A}\left(Q^{2}\right) ; G_{M}^{s}\left(Q^{2}\right)=\mu_{s} G_{D}^{V}\left(Q^{2}\right) \\
G_{E}^{s}\left(Q^{2}\right)=\rho_{s} Q^{2} / 4 M^{2} G_{D}^{V}\left(Q^{2}\right) ; \quad \text { with } G_{D}^{V(A)}\left(Q^{2}\right)=1 /\left(1+Q^{2} / M_{V(A)}^{2}\right)^{2}
\end{gathered}
$$

while the strengths $g_{A}^{s}, \mu_{s}$ and $\rho_{s}$ are taken as free parameters. The same values for the strange cutoff masses as for the non-strange vector (axial) form factors are assumed.

In Fig. 1(a) the ratios $R_{\nu}$ and $R_{\bar{\nu}}$ versus $\mu_{s}$ are shown for two values of the axial-strange constant: $g_{A}^{s}=0,-0.15$ and three values of the electric strange constant: $\rho_{s}=0, \pm 2$. The axial cutoff mass is $M_{A}=1.032 \mathrm{GeV}$. As it is seen from the same Fig. 1 (a), a value of the strange axial constant $g_{A}^{s}$ as large as -0.15 is not favoured by the BNL-734 data.

Results of the calculation of the ratio $R$ and the integral asymmetry $\left\langle\mathcal{A}_{p}\right\rangle$ are shown in Fig. $1(\mathrm{~b})$ : both for $R$ and $\left\langle\mathcal{A}_{p}\right\rangle$ the effects induced by the axial and magnetic strange form factors are similar. These effects are clearly larger (in $R$ ) than the ones due to the electric strange form factor. Moreover it is worth noticing that the integral asymmetry does not depend at all upon the electric strange form factor. All the considered values of the strange parameters are compatible with the asymmetry $\left\langle\mathcal{A}_{p}\right\rangle$ within the experimental errors. However for values of $g_{A}^{s}$ as large as -0.15 the experimental value of $R$ favours $\mu_{s} \leq 0$.

The experimental uncertainties are of the same order as the effects of the strange form factors of the nucleon. Keeping this in mind and without any claim for a definitive evidence, the results seem to favour negative values of the magnetic strange parameter, $\mu_{s}$, if $-g_{A}^{s}$ is relatively large.

The value of the strange magnetic form factor of the nucleon has been recently measured at BATES, with the result $G_{M}^{s}\left(0.1 \mathrm{GeV}^{2}\right)=0.23 \pm 0.37 \pm$ $0.15 \pm 0.19$. This value is affected by large experimental and theoretical uncertainties (the last error refers to the estimate of radiative corrections), but it is centered around a positive $\mu_{s}$, although it is still compatible with zero or negative values of $\mu_{s}$. Let me also notice that, if the $\mathrm{P}$-odd asymmetry 

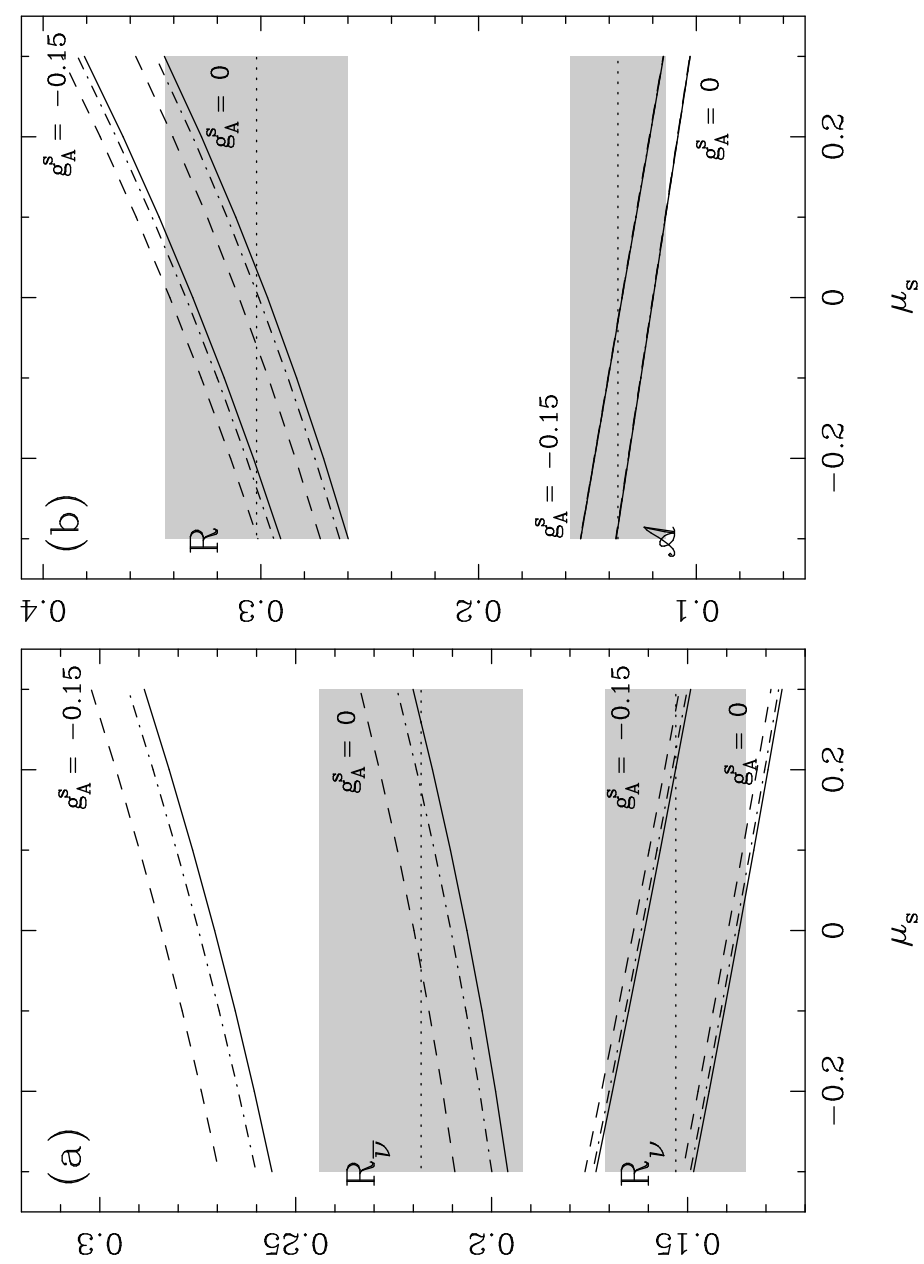

Figure 4. The ratios $R_{\nu}$ and $R_{\bar{\nu}}$ (a), and $R$ and $\left\langle\mathcal{A}_{p}\right\rangle$ (b), as a function of $\mu_{s}$ : all curves correspond to $\nu(\bar{\nu})-$ p elastic scattering. Results are shown for $g_{A}^{s}=0$ and $g_{A}^{s}=-0.15$. In both cases we have chosen $\rho_{s}$ to be: $\rho_{s}=0$ (solid line), $\rho_{s}=-2$ (dot-dashed line) and $\rho_{s}=+2$ (dashed line). The shadowed regions correspond to the experimental data measured at BNL-734 experiment. 
measured in the scattering of polarized electrons on nucleons will provide a more stringent information on the strange magnetic form factor, then future, precise experiments combining the measurement of $\nu$ and $\bar{\nu}$-proton scattering could allow a determination of the axial strange form factor and of the electric one.

One can conclude that the uncertainty of the available data does not allow to set stringent limits on the strange vector and axial-vector parameters, but future, more precise measurements could make their determination possible in a model independent way.

\subsection{Parity violating electron scattering and target asymmetry}

As already mentioned in the previous section, a first measurement of the PV beam asymmetry $\left(\mathcal{A}_{L R}\right)$ in $\vec{e}-p$ elastic scattering was performed at Bates/MIT Laboratory by the SAMPLE Collaboration giving the first experimental determination of the proton strangeness magnetic form factor at $Q^{2}=0.1(\mathrm{GeV} / \mathrm{c})^{2}\left(\mu_{s}=0.23 \pm 0.37 \pm 0.15 \pm 0.19 \mu_{N}\right)$.

Because of the difficulties inherent in the PV electron scattering experiment an independent determination of $\mu_{s}$, of the strangeness radius $r_{s}^{2}$ and of other strangeness properties of the proton could be extremely useful.

Recently Moscani et al 11 reported on the results of a study on the asymmetry $\mathcal{A}$ of the elastic $e-\vec{p}$ scattering cross section (in the low $Q^{2}$ range) arising from the polarization of the proton target. In principle, this asymmetry is even more versatile than $\mathcal{A}_{L R}$ for disentangling the different weak form factors because the polarization of the proton target can be freely chosen whereas the electron beam can be polarized only along the beam momentum.

The only nonzero components of the target asymmetry are those in the scattering plane, i.e. the transverse $\left(\mathcal{A}_{x}\right)$ and the longitudinal $\left(\mathcal{A}_{z}\right)$ ones (we assume the z-axis along the momentum transfer).

In Fig. 5 the angular distribution of $\mathcal{A}_{x}, \mathcal{A}_{z}$ and of the modulus of $\mathcal{A}_{L R}$ are shown for $Q^{2}=0.1(\mathrm{GeV} / \mathrm{c})^{2}$, calculated with Jaffe's value $r_{s}^{2}=0.16 \mathrm{fm}^{2}$ and the central experimental value $\mu_{s}=0.23 \mu_{N}$. $\mathcal{A}_{x}$ and $\mathcal{A}_{z}$ show a remarkably different angular dependence as $\vartheta_{e^{\prime}}$ increases: $\mathcal{A}_{x} \rightarrow 0$ at backward angles while $\mathcal{A}_{z}$ reaches its maximum.

$\mathcal{A}_{z}$ does not depend on $\tilde{G}_{E}$ (and then on $G_{E}^{(s)}$ ) and its dependence on $\tilde{G}_{A}$ is lowered with respect to that on $\tilde{G}_{M}$ because $g_{V}^{e} \ll g_{A}^{e}$, in particular at backward angles. In principle, a measurement of $\tilde{G}_{M}$ in the target asymmetry is more convenient than in the helicity asymmetry making $\mathcal{A}_{z}$ an useful quantity for a determination of $\mu_{s}$ complementary to that coming from $\mathcal{A}_{L R}$. Then, a determination of $\tilde{G}_{A}$ (and of $g_{A}^{(s)}$ ), alternative to that deriving from 


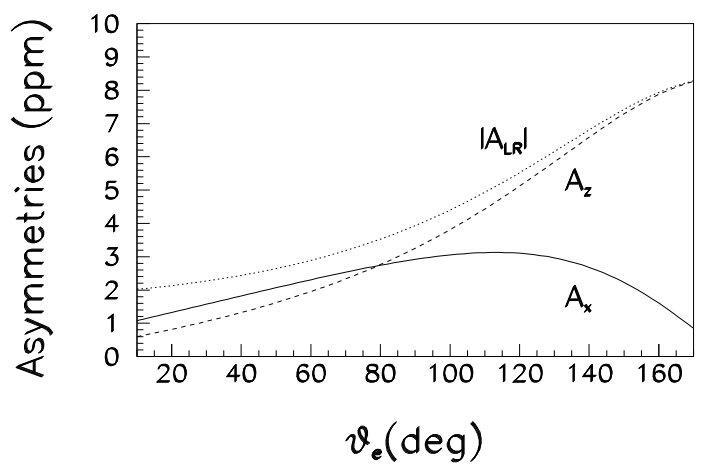

Figure 5. Angular distribution of the target asymmetry $\mathcal{A}_{x}\left(\vartheta_{e^{\prime}}\right)$ (full line), $\mathcal{A}_{z}\left(\vartheta_{e^{\prime}}\right)$ (dashed line) and of the modulus of the helicity asymmetry $\mathcal{A}_{L R}\left(\vartheta_{e^{\prime}}\right)$ (dotted line) at $Q^{2}=0.1(\mathrm{GeV} / \mathrm{c})^{2}$, with $\mu_{s}=0.23 \mu_{N}$ and $r_{s}^{2}=0.16 \mathrm{fm}^{2}$.

$\nu / \bar{\nu}$ scattering experiments could be carried out in this kind of PV electron scattering experiments.

In conclusion:

i) The asymmetry $\mathcal{A}$ of the elastic $e-\vec{p}$ scattering cross section arising from the polarization of the proton target may be a possible PV observable for an experimental determination of the proton weak form factors.

ii) The most convenient decomposition of $\mathcal{A}$ is obtained considering the proton polarization along and perpendicular to the momentum transfer.

iii) The longitudinal asymmetry $\mathcal{A}_{z}$ is independent of $\tilde{G}_{E}$ allowing an experimental determination of the proton strangeness magnetic moment $\mu_{s}$.

iv) The transverse asymmetry $\mathcal{A}_{x}$ is rather sensitive to the proton strangeness radius $r_{s}^{2}$ in the case of backward detected electrons.

v) A peculiarity of $\mathcal{A}_{x}$ and $\mathcal{A}_{z}$ with respect to $\mathcal{A}_{L R}$, is that their dependence on $\tilde{G}_{A}$ can be enhanced over that on $\tilde{G}_{E}, \tilde{G}_{M}$. In fact, in the strict forward scattering $\left(\vartheta_{e^{\prime}}=0^{\circ}\right) \mathcal{A}_{x}$ and $\mathcal{A}_{z}$ are determined by $\tilde{G}_{A}$ only.

\section{Deep inelastic scattering}

Constituent quark models, on one side, and the parton picture, on the other side, represent two complementary descriptions of the hadron structure and 
the birth of QCD set the general framework to understand deep inelastic scattering (DIS) beyond the parton model. In the recent past a lot of work has been devoted to the attempt of reproducing the experimental deep inelastic structure functions at high momentum transfer starting from a parton parametrization at a low resolution scale $Q_{0}^{2}$ where the valence contribution becomes dominant. In this way quark models, summarizing a great deal of hadronic properties, may substitute low-energy parametrizations. Following such a path, a partonic description can be generated from gluon radiation even off a purely valence quark system, which can be used to generate the non perturbative input occurring in the Operator Product Expansion (OPE) analysis of lepton-hadron scattering in QCD.

In the next sections I summarize some of the work done in this field by different authors.

\subsection{Exact models}

In the parton model of DIS, it is assumed that the final state interaction (FSI) of the struck parton with the remnants of the target is a higher twist effect, i.e., an effect which is suppressed at least as $m^{2} / Q^{2}$. The qualitative motivation of this assumption is that the time needed for the absorption of the virtual photon by the struck quark is much smaller than the time of its hadronization and therefore in the process of absorption the struck quark can be considered as approximately free.

Of course the relevance of the FSI has to be studied in the framework of nonperturbative QCD, but in absence of a full solution it is desirable to consider models in which the structure functions can be calculated exactly and therefore it is possible to check whether the FSI is indeed a higher twist effect.

Pace et al 12 investigated the role played by FSI in DIS for an exactly solvable relativistic quark model, within the light-cone (= front-form) hamiltonian dynamics. The confinement can be ensured by choosing a quark-quark potential such that the mass operator of a system with a fixed number of relativistic constituent quarks has only the discrete spectrum (while in QCD confinement is understood as the property of the quark and gluon Green functions to have no poles for real values of the mass). The purpose is to verify whether the naive treatment of confinement in relativistic CQMs is compatible with the parton model.

The authors consider a simple system composed by two relativistic particles interacting via the relativistic harmonic oscillator potential. The electromagnetic current matrix elements exhibit the correct properties under

final: submitted to World Scientific on November 13, 2018 
Poincaré transformations and fulfill the current conservation. In the proper Breit frame, the relevant components of the current are the same as in the parton model. Then, in the framework of the light-cone hamiltonian dynamics, one can derive exact expressions for the DIS structure functions, including, for the first time, the FSI effects calculated exactly in a relativistic model.

Their results can be summarized in the following way:

i) the relativistic calculation differs from the nonrelativistic ones considered in in several aspects. In particular, the Bjorken limit implies that one gets a finite contribution to the structure functions only from excited states with $n \rightarrow \infty$, while the nonrelativistic approach is valid only if $n \ll\left(m_{0} / a\right)^{2}$. Furthermore in the infinite momentum frame only the transverse components of the hadronic tensor survive in the Bjorken limit, while in the nonrelativistic case the component $W^{00}$ is the dominant one.

ii) The results could be considered as an argument in favor of the "common wisdom", according to which the FSI in the Bjorken limit is a higher twist effect.

iii) The choice of the current is compatible with Poincaré invariance and current conservation. However, these requirements do not determine the current operator uniquely and many body components could be present in $J^{\mu}(0)$. Therefore one should study whether the results of the parton model can still be recovered in the Bjorken limit if the operator $J^{\mu}(0)$ contains many-body interaction terms.

\subsection{Covariance and DIS}

It is quite evident that relativistic effects to the nucleon wave function, as well as, covariance requirements, are needed even for a phenomenological description of the structure of hadrons. To this aim I discuss a constituent quark model approach based on a light-front realization of the Hamiltonian dynamics and recent applied for the calculation of both polarized and unpolarized parton distributions 13.13.

The parton distributions at the hadronic scale are assumed to be valence quarks and gluons, and their twist two component is determined by the quark momentum density.

The relevant effects of the relativistic covariance are particularly evident looking at the polarized distributions. In that channel the introduction of Melosh transformations results in a substantial suppression of the responses at large values of $x$ and in an enhancement of the response for $x \leq 0.15$. The consequences can be appreciated looking at the results at the experimental scale after a Next-to-Leading order evolution (see Fig. 6) and to the orbital 


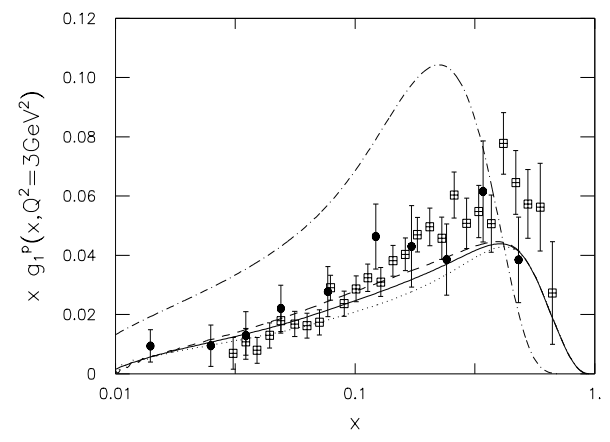

Figure 6. The proton polarized structure function $g_{1}$ at $Q^{2}=3 \mathrm{GeV}^{2}$. The full curve represents the NLO $(\overline{M S})$ results of a complete light-front calculation within a scenario where no gluons are present at the hadronic scale (scenario A); the corresponding nonrelativistic calculation are shown by the dot-dashed line. A scenario including negative polarized gluons $\left(\int \Delta G=-0.7\right)$ at the hadronic scale is summarized by the dotted line; dashed line shows the case of positive gluon polarization $\left(\int \Delta G=+0.7\right)$.

angular momentum parton distributions 15 . The Melosh rotation dynamics introduce the basic new ingredient in the calculations and its effect is quite sizeable in suppressing the proton response in the region $x \leq 0.4$.

\subsection{Relativistic spin effects in Drell-Yan processes}

A complete description of the spin degrees of freedom of quarks and antiquarks in the nucleon requires, at leading twist, the definition of two sets of parton distributions. One of them, the helicity distribution $g_{1}\left(x, Q^{2}\right)$, have been intensively investigated in the last few years while the so called transversity distribution, $h_{1}\left(x, Q^{2}\right)$, has come to the attention of theorists and experimentalists more recently in the analysis of Drell-Yan spin asymmetries. In fact transversity is strongly suppressed (by powers of $m_{q} / Q$ ) in deep inelastic lepton-nucleon scattering and in general in any hard process that involves only one parton distribution. In hadron-hadron collisions the chirality of the partons that annihilate is uncorrelated and the previous restrictions do not apply.

It is rather well known that at the hadronic scale the equality $h_{1}\left(x, Q_{0}^{2}\right)=$ $g_{1}\left(x, Q_{0}^{2}\right)$ is a typical outcome of non-relativistic models of the nucleon, in which motion and spin observables are uncorrelated. In other words, any departure from the previous identity is a signature of relativity in the employed 
hadronic model. A complete theoretical study of $h_{1}$ and $g_{1}$ has to account for both: the relativistic effects which distinguish $h_{1}$ from $g_{1}$ at the nonperturbative scale, and the pQCD evolution which differs for the two structure functions.
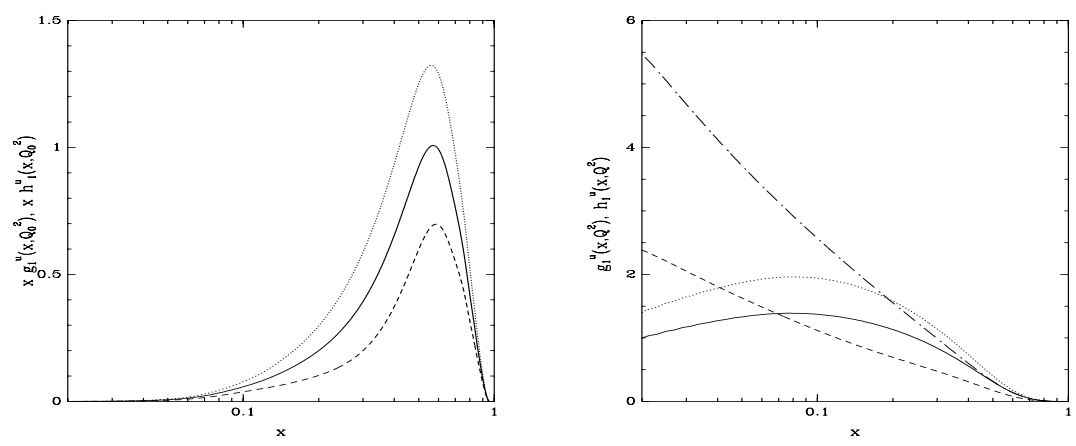

Fig. 6bis. Helicity and transversity distributions for the $u$ quark at the hadronic scale $Q_{0}^{2}=0.094 \mathrm{GeV}^{2}$ (left panel) and after evolution up to $Q^{2}=100 \mathrm{GeV}^{2}$ (right panel). On the left the solid line corresponds to $x h_{1}$, the dashed line to $x g_{1}$ and the dotted line is the result when Melosh rotation is not considered $\left(h_{1}=g_{1}\right)$. On the right the solid and dashed lines represent $h_{1}$ and $g_{1}$ respectively. The dotted and dash-dotted lines correspond to $h_{1}$ and $g_{1}$ when Melosh Rotation is neglected.

A quantitative study of the relativistic effects in $h_{1}$ and $g_{1}$ due to the correlations of spin and parton motion in the hadronic systems has been recently completed 14 . It makes use of the light-front approach previously discussed and the interplay between motion and spin is made explicit through the Melosh rotations. The light-front covariant quark model is used to compute the leading twist contribution to the matrix elements at the hadronic scale $Q_{0}^{2}$. The non-perturbative input is then evolved, at NLO, up to a higher $Q^{2}$ scale.

In Fig. 6bis the results for $h_{1}^{u}$ and $g_{1}^{u}$ at the hadronic scale $Q_{0}^{2}$ (left panel) and at the partonic scale $Q^{2}=100 \mathrm{GeV}^{2}$ (right panel) are shown. A remarkable difference between $x h_{1}\left(x, Q_{0}^{2}\right)$ and $x g_{1}\left(x, Q_{0}^{2}\right)$ appears at large $x$, reaching a peak at $x \approx 0.5$. Quantitatively they are bigger that those obtained within bag models. It is clear that the probability of transverse polarization is larger than the longitudinal one when relativistic effects are considered. The results obtained neglecting relativistic spin-motion correlation (induced in the lightcone approach by the Melosh rotations) are shown also in the same figure. One gets $h_{1}\left(x, Q_{0}^{2}\right)=g_{1}\left(x, Q_{0}^{2}\right)$ as expected. After evolution $g_{1}$ and $h_{1}$ differ 


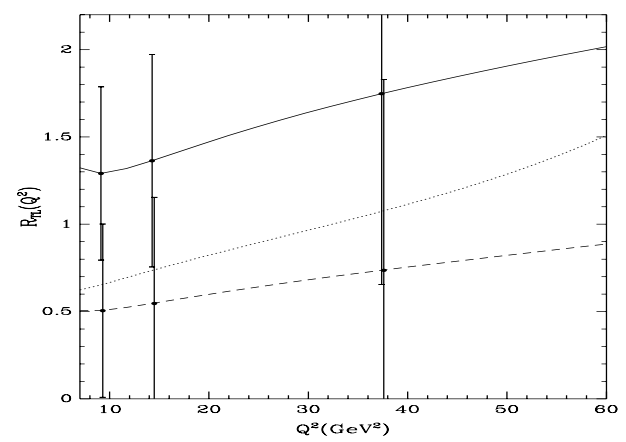

Figure 7. Ratio between transverse and longitudinal parton distributions, Eq. 15), as a function of the invariant mass of the produced lepton pair $\left(Q^{2}\right)$ at a center of mass energy corresponding to HERA $-\vec{N}(\sqrt{s}=39.2) \mathrm{GeV}$. Dashed line shows results neglecting Melosh rotations, the dotted line corressponds to the non-realtivistic model. Error bars have been calculated at LO and include acceptance corrections. Error bars in the lower curve have been slightly shifted to appreciate the overlap.

mainly at low $x(x \leq 0.1)$ because of pQCD evolution, while the inclusion of the correlations between spin and motion produce large effects also in the medium and large $x$ region.

In order to look for relativistic spin effects, a specific observable has been defined in ref.14, namely the ratio

$$
R_{T L}\left(Q^{2}\right)=\frac{\int\left(\sum_{a} e_{a}^{2} h_{1}^{a}\left(x_{1}, Q^{2}\right) h_{1}^{\bar{a}}\left(x_{2}, Q^{2}\right)+\left(x_{1} \leftrightarrow x_{2}\right)\right) d y}{\int\left(\sum_{a} e_{a}^{2} g_{1}^{a}\left(x_{1}, Q^{2}\right) g_{1}^{\bar{a}}\left(x_{2}, Q^{2}\right)+\left(x_{1} \leftrightarrow x_{2}\right)\right) d y}
$$

where $g_{1}^{a}\left(x, Q^{2}\right)\left(h_{1}^{a}\left(x, Q^{2}\right)\right)$ are the lngitudinally (transverse) polarized parton distributions with flavor $a$ and charge $e_{a}$; the arguments $x_{1}$ and $x_{2}$ are related, for Drell-Yan processes, to the center of mass energy $\sqrt{s}$, the invariant mass of the produced lepton pair $Q^{2}$, and the rapidity $y=\arctan \left(Q^{3} / Q^{0}\right): x_{1}=$ $\sqrt{Q^{2} / s} e^{y}$ and $x_{2}=\sqrt{Q^{2} / s} e^{-y}$.

The results for this ratio and for the kinematics of HERA $-\vec{N}$ are shown in Fig. T The relative insensitivity to the details of the chosen potential is also evident in this representation. In the error bars shown take into account the limited acceptance of the detectors. While a measurement in the region $Q>5 \mathrm{GeV}$ cannot distinguish the importance of Melosh Rotations, in the low mass region $(Q \approx 3 \mathrm{GeV})$ it would be possible to single out which is the right spin-flavor basis, though some overlap between the error bars still persists. For RHIC the acceptance corrections are too large to appreciate the differences. 


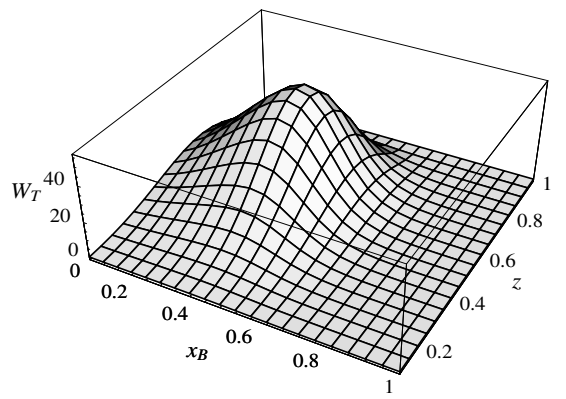

Figure 8. Dependence of the structure function $W_{T}^{[\mathrm{UU}]}$ on $x_{B}$ and $z$ at $P_{h \perp}=0$.

\subsection{Semi-inclusive structure functions}

Detecting ond 18 or two 17 of the hadrons produced in the high-energy scattering process, one is sensitive not only to the distribution of partons inside the target hadron, but also to the mechanism of hadronization, through which a quark gives rise to a jet of new hadrons. One is then able to measure not only distribution functions, but also the so-called fragmentation functions. These functions are presently considered to be very interesting and their experimental measurement is in progress (HERMES, COMPASS, RHIC). Neither the distribution nor the fragmentation functions can be calculated from first principles within perturbative QCD, because they belong to the non-perturbative realm of bound states and models are required.

In this context, model evaluations of the structure functions can be again useful. The spectator model proved to be in qualitative agreement with the known (transverse momentum integrated) distribution and fragmentation functions evolved at low energies. Therefore, one expects it to give reasonable estimates for the convolution integrals in semi-inclusive DIS. A good example 16 is given in Fig. 8 for the reaction $e p \rightarrow e^{\prime} \Lambda X$ for unpolarized proton target and unpolarized produced $\Lambda$.

The basic assumption of the spectator model is that the target hadron can be divided into a quark and an effective spectator state with the required quantum numbers, which is treated to a first approximation as being on-shell with a definite mass. In the case of a baryon target, this second particle is a diquark. The quark fragments into a jet, from which one hadron is 
eventually detected; the remnants of the jet are treated effectively as an onshell spectator state. If the detected hadron is a baryon, the second particle is an anti-diquark. The vertex coupling the baryon to quark and diquark includes a form factor preventing the quark from being far off-shell. The large $p^{2}$-behavior of the form factor is controlled by a parameter.

An important feature of the analysi 16 is the dependence of the crosssections on the transverse momentum of the outgoing hadron, $P_{h \perp}$. The measurement of this variable gives access to two new contributions to the structure functions which have never been observed so far, because they vanish if the cross-section is integrated over $P_{h \perp}$. Furthermore, the dependence of the cross-section on $P_{h \perp}$ indirectly tests the distribution of partonic transverse momentum inside the hadron: a distribution largely unknown at the present.

\section{Concluding remarks}

In the present overwiev I shortly summarized some of the italian research activities on the field of hadronic degrees of freedom since the last two years. It seems to me that the contribution of the nuclear physics community to the topic contains few specific aspects that are peculiar of a long tradition in studying complex systems. One of them is the systematic use of many-body techniques in implementing constituent quark models both non-relativistic and relativistic. The results are relevant for the interpretation of the experimental data at the TJNAF and other accelerator facilities in the GeV region (e.g. MAMI) as illustrated by Taiuti and Pacati in their talks 18.19 .

A second example is well illustrated by the study of the electroweak structure of the nucleon: basic quantities like the strange content of the nucleon, can get new insights by using the nucleus system as a filter to select interesting observables. Addional examples can be found in the study of deep inelatic scattering. Basic questions related to the complex dynamics of confinement and/or final state interaction can be investigated by means of sophysticate approaches which belong to the nuclear physics knowhow. In particular the study of the (largely) unknown non-perturbative part of the Operator product expansion approach to deep inelastic hadronic physics, is receiving important contributions from our community and they are relevant for the experiments at CERN, RHIC and HERA.

\section{Acknowledgments}

During the preparation of this contribution my wife, after a long illness, died. I dedicate this paper to her memory asking the reader for a prayer.

final: submitted to World Scientific on November 13, 2018 


\section{References}

1. G. Morpurgo, La Rivista del Nuovo Cimento 22,1 (1999).

2. G. Dillon and G. Morpurgo, Phys. Lett. B448, 107 (1999); B481, 239 (1999); B485, 429 (1999).

3. M. Aiello, M.M. Giannini and S. Santopinto, J. Phys. J 24 (1998) 753.

4. M. De Sanctis, E. Santopinto, M.M. Giannini, Eur. Phys. J. A1 (1998) 187.

5. D. Drechsel, M. Vanderhagaen, M.M. Giannini and E. Santopinto, Phys. Lett. B484, 236 (2000).

6. E. Pace, G. Salmé, F. Cardarelli and S. Simula, Nucl. Phys. A666-667, 33 (2000).

7. F. Cardarelli, E. Pace, G. Salmé and S. Simula, Few-Body Syst. Suppl. 10, 407 (1999); 11, 66 (1999).

8. R. F. Wagenbrunn, S. Boffi, W. Klink, W. Plessas and M. Radici, nuclth/0010048.

9. W.M. Alberico, M.B. Barbaro, S.M. Bilenky, J.A. Caballero, C. Giunti, C. Maieron, E. Moya de Guerra and J.M. Udias, Nucl. Phys. A651, 277 (1999).

10. W.M. Alberico, M.B. Barbaro, S.M. Bilenky, J.A. Caballero, C. Giunti, C. Maieron, E. Moya de Guerra and J.M.Udias, Phys. Lett. B438, 9 (1998).

11. M. Moscani, B. Mosconi and P. Ricci, Phys. Rev. C 59, 2844 (1999).

12. E. Pace, G. Salmé and F.M. Lev, Phys. Rev. C 57 (1998) 2655.

13. P. Faccioli, M. Traini and V. Vento Nucl. Phys. A656 (1999) 400;

14. F. Cano, P. Faccioli and M. Traini, Phys. Rev. D 62, 094018 (2000).

15. F. Cano, P. Faccioli, S. Scopetta and M. Traini, Phys.Rev. D 62 (2000) 054023 .

16. A. Bacchetta, S. Boffi and R. Jakob, submitted to Eur. Phys. J. C; hep-ph/0003243.

17. A. Bianconi, S. Boffi, R. Jakob and M. Radici, Phys. Rev. D 62, 034008 (2000); 62, 034009 (2000).

18. M. Taiuti, these proceedings.

19. F. Pacati, these proceedings. 